\title{
ESTIMATIVA DO CUSTO DE IMPLANTAÇÃO DA AGRICULTURA IRRIGADA, UTILIZANDO O SISTEMA DE INFORMAÇÃO GEOGRÁFICA ${ }^{1}$
}

\author{
DANIEL F. DE CARVALHO ${ }^{2}$, WILSON A. DA SILVA ${ }^{3}$, MARCOS B. CEDDIA ${ }^{4}$, \\ ELMO L. X. TANAJURA ${ }^{5}$, ANDRÉ L. O. VILLELA ${ }^{6}$
}

\begin{abstract}
RESUMO: A proposta deste trabalho foi utilizar o Sistema de Informações Geográficas (SIG) com o objetivo de indicar áreas agroeconomicamente mais aptas para a implantação da cultura do coqueiro irrigado. Foram utilizadas informações referentes ao assentamento rural Antonio Farias, localizado no município de Campos dos Goytacazes, região Norte do Estado do Rio de Janeiro. O software IDRISI 32 gerou informações que técnicos, extensionistas e produtores rurais podem utilizar no planejamento da agricultura em áreas irrigadas. Foram utilizados mapas digitais de solo e planialtimetria, além de mapas temáticos com informações de drenagem, estradas e limite dos lotes. A partir de operações de sobreposição dos temas solo, clima e relevo, foram criados novos mapas temáticos, que mostram os gastos parciais para implantar 1 ha irrigado da cultura do coqueiro-anão. $O$ gasto de implantação foi composto pela soma dos desembolsos para a compra de calcário, fertilizantes, tubulação e motobomba. A partir dos mapas criados, foram observadas variações expressivas nos desembolsos para implantação de 1 ha de coqueiro irrigado entre os lotes do assentamento. Foi possível observar que a metodologia utilizada se mostrou eficaz na identificação das áreas mais aptas, sob o ponto de vista agroeconômico, para a implantação de culturas irrigadas, sendo facilmente aplicável para outras culturas e áreas de estudo.
\end{abstract}

PALAVRAS-CHAVE: planejamento da irrigação, mapas de custo de implantação, SIG.

\section{ESTIMATIVE IMPLANTATION COST OF IRRIGATED AGRICULTURE, USING GEOGRAPHICAL INFORMATION SYSTEM}

\begin{abstract}
The proposal of this study was to use the Geographical Information System (GIS) in order to indicate the best areas to implant irrigated crop. It was used information of the rural settlement area of Antonio Farias, localized in Campos dos Goytacazes municipality, north region of Rio de Janeiro State, Brazil. Using the software IDRISI 32, it was possible to create information to support crop irrigation planning by technicians, extensionists and farmers. Digital maps of the soil and topographic maps, as well as thematic maps of drainage, roads and farm boundaries were used. The maps of costs per hectare of implementing irrigated crops of dwarf coconut tree were obtained by crossing soil, climate and relief data. The final cost in this study is composed by the sum of partial costs of liming, fertilization, irrigation pipes and pump. It was observed from the generated maps that the implantation cost of irrigation varied between settlement parcels (as some were in favorable areas). The proposed methodology was able to identify the most agroeconomically apt areas for implementing crop irrigation systems. This methodology used in this study can also be extended to analyze irrigation implementation costs for other crops just by changing the agronomic indices for other crops.
\end{abstract}

KEYWORDS: irrigation planning, implantation cost maps, GIS.

\footnotetext{
${ }^{1}$ Extraído da Dissertação de Mestrado do segundo autor. Projeto financiado pela FAPERJ.

${ }^{2}$ Prof. Adjunto, DE/IT/UFRRJ, Seropédica - RJ, Fone: (0XX21) 2682-1865, carvalho@ufrrj.br. Bolsista do CNPq.

${ }^{3}$ Mestre em Fitotecnia, Doutorando em Ciência do Solo, UFRRJ, Seropédica - RJ, wilson008@ bol.com.br.

${ }^{4}$ Prof. Adjunto, Departamento de Solos, Instituto de Agronomia, UFRRJ, Seropédica - RJ, ceddia@ufrrj.br

${ }^{5}$ Estudante de Engenharia de Agrimensura, Bolsista de Iniciação Científica, PIBIC/UFRRJ/CNPq.

${ }^{6}$ Licenciado em Ciências Agrícolas, Prof. Substituto, Departamento de Arquitetura e Urbanismo, UFRRJ.

Recebido pelo Conselho Editorial em: 3-6-2003

Aprovado pelo Conselho Editorial em: 25-5-2005
} 


\section{INTRODUÇÃO}

O tratamento de informações geográficas espaciais é hoje um requisito necessário para controlar e ordenar a ocupação das unidades físicas do meio ambiente, pressionadas por decisões que invariavelmente se contrapõem a uma lógica racional de seu uso. Para acompanhar o ritmo veloz e a complexidade dessa ocupação e utilização do solo, é preciso dispor de técnicas que provejam referências espaciais da ordenação dessa ocupação, passíveis de tratamentos automatizados. De acordo com ASSAD \& SANO (1998), os Sistemas de Informações Geográficas (SIG) manipulam dados de diversas fontes e formatos, dentro de um ambiente computacional ágil e capaz de integrar as informações espaciais temáticas e gerar novos dados derivados dos originais. BURROUGH (1990) define SIG como sendo um conjunto de ferramentas de coleta, armazenamento, recuperação, transformação e apresentação dos dados do mundo real para um objetivo específico; esse objetivo específico, de modo geral, é o apoio à tomada de decisões.

No setor agrícola, ASSAD \& SANO (1993) já chamavam a atenção para futuras aplicações da tecnologia SIG, como sua utilização em trabalhos de fertilidade de solos, mecanização agrícola e na estimativa da produtividade agrícola. Atualmente, o uso de informações geográficas na agricultura tem-se mostrado como ferramenta importante no planejamento de atividades, sendo possível criar de forma prática e menos onerosa, bancos de dados e documentos cartográficos, como mapas temáticos, com as mais diversas informações que servirão de base para um bom planejamento de uma atividade, como, por exemplo, o planejamento de áreas irrigadas.

Ao se planejar um sistema de irrigação, são necessários estudos preliminares que forneçam informações visando à comprovação da sua viabilidade técnica e econômica. Tais estudos devem ser compostos de informações gerais sobre a área a ser irrigada, sobre o meio físico (topografia, pedologia, características hidro-físico-químicas, climatologia, disponibilidade hídrica, etc.) e aspectos socioeconômicos. Portanto, um dos primeiros passos para o dimensionamento de sistemas de irrigação, sejam eles pressurizados ou por superfície, é a coleta de dados de solo, planta, clima e topográficos. Embora seja essa uma tarefa muitas vezes trabalhosa, é fundamental que um planejamento global seja realizado de modo eficiente, tanto pelo fato de esses empreendimentos muitas vezes requererem altos investimentos, quanto para minimizar a ocorrência de erros de projeto e insucessos na obtenção do retorno econômico. SIFUMA et al. (2000) realizaram estudos sobre planejamento da irrigação em sistemas agrícolas e concluíram que existe complexa interação de fatores (climáticos, requerimento hídrico e preferência dos produtores), afetando a seleção de um sistema produtivo e também que um bom planejamento do calendário agrícola pode reduzir o requerimento de água e maximizar os benefícios do projeto de irrigação.

Independentemente do método e das culturas instaladas, um planejamento adequado de áreas irrigadas deve efetivamente estar integrado com as informações de clima, de solo e também com as funções de resposta das culturas em relação à lâmina d'água aplicada, além de recursos de capital, de trabalho e de tecnologia agrícola, mantendo as condições de umidade e nutrientes no solo favoráveis ao bom desempenho da cultura (BOWMAN et al., 1991). Na maioria dos casos, as incertezas em torno dessas informações dificultam as decisões de planejamento e manejo da irrigação. Porém, é possível resolver esse problema, utilizando-se dos SIGs, que permitem a realização de análises complexas ao integrar dados de diversas fontes e ao criar bancos de dados georreferenciados a respeito da área de interesse.

Neste estudo, pretendeu-se testar a hipótese de que os Sistemas de Informações Geográficas podem ser utilizados de maneira eficiente na indicação de áreas mais aptas para a implantação de culturas irrigadas, baseando-se em características econômicas e agroedafoclimáticas de um assentamento rural, no norte do Estado do Rio de Janeiro. A obtenção dessas informações visam a 
auxiliar os técnicos, extensionistas e produtores rurais no planejamento da agricultura em áreas irrigadas, utilizando-se de técnicas de geoprocessamento.

\section{MATERIAL E MÉTODOS}

\section{Localização da área de estudo}

Para a realização deste estudo, visando à estimativa do custo de implantação da agricultura irrigada, foi utilizada área localizada no município de Campos dos Goytacazes - RJ, constituindo no assentamento rural Antônio Farias. Essa área está localizada entre as coordenadas geográficas $21^{\circ} 00^{\prime} \mathrm{e}$ $22^{\circ} 00^{\prime}$ de latitude sul e $41^{\circ} 00^{\prime}$ e $42^{\circ} 00^{\prime}$ de longitude oeste (Figura 1).

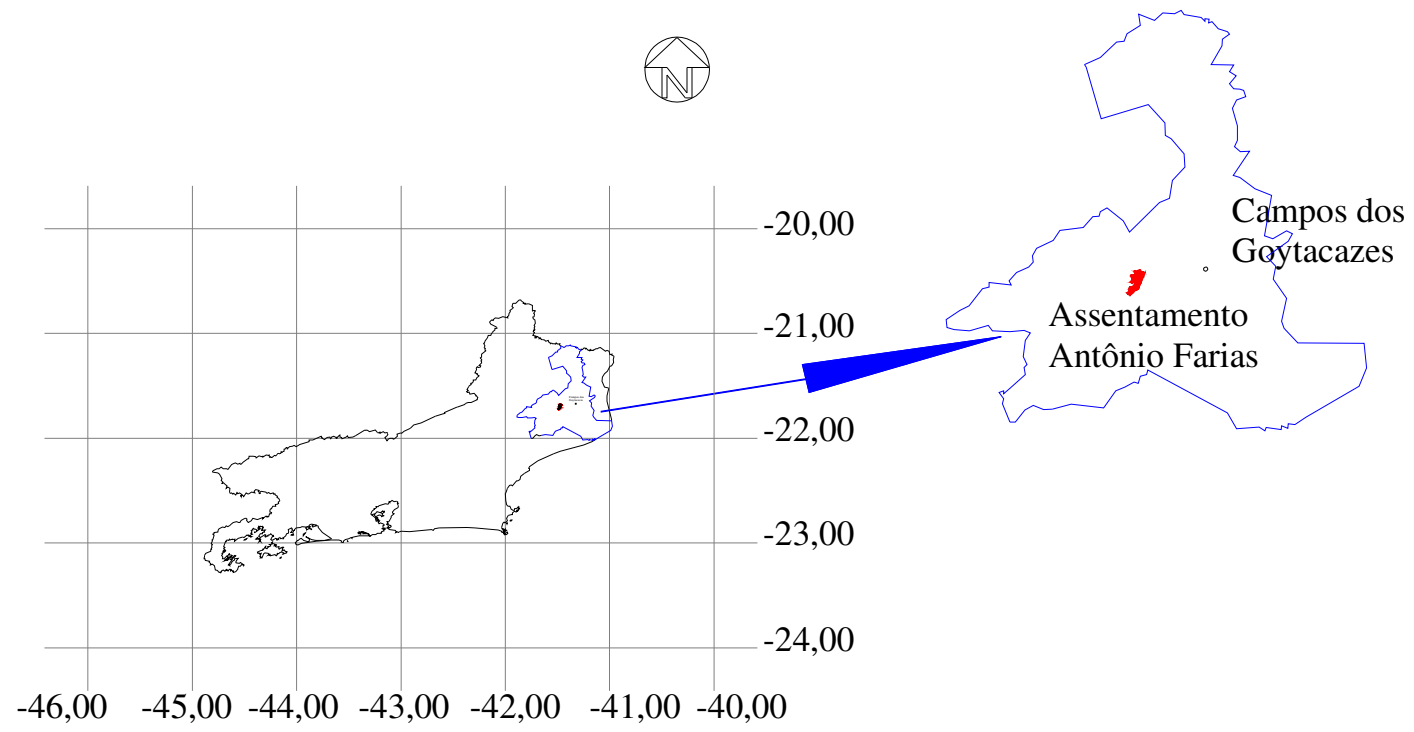

FIGURA 1. Localização do Assentamento Antônio Farias.

No assentamento Antônio Farias, existem nove núcleos de irrigação, preestabelecidos pelo ITERJ - Instituto de Terras e Cartografia do Estado do Rio de Janeiro (Figura 2). O estudo foi realizado para cada núcleo separadamente, sendo considerada como área útil do núcleo aquela referente à soma total das áreas de todos os lotes.

\section{Estimativa da lâmina de irrigação e da vazão de projeto}

Para o cálculo da lâmina de projeto ou demanda de irrigação, foram utilizados os dados climáticos de série histórica referente à estação Campos dos Goytacazes, cadastrado no INMET como 83698. Os arquivos de dados foram organizados e, utilizando o programa computacional DEMANDA, desenvolvido por CARVALHO et al. (1999), foi estimada a lâmina diária de evapotranspiração de referência (ETo), por meio da equação de Penman-Monteith (SMITH, 1991).

O estudo foi desenvolvido para a cultura do coqueiro (Cocos nucifera L.), por apresentar grande potencial para a região, uma vez que a mesma vem recebendo importantes subsídios do Governo do Estado por meio do projeto FRUTIFICAR, como forma de incentivar esse tipo de atividade agrícola, principalmente entre os pequenos e médios produtores rurais. 


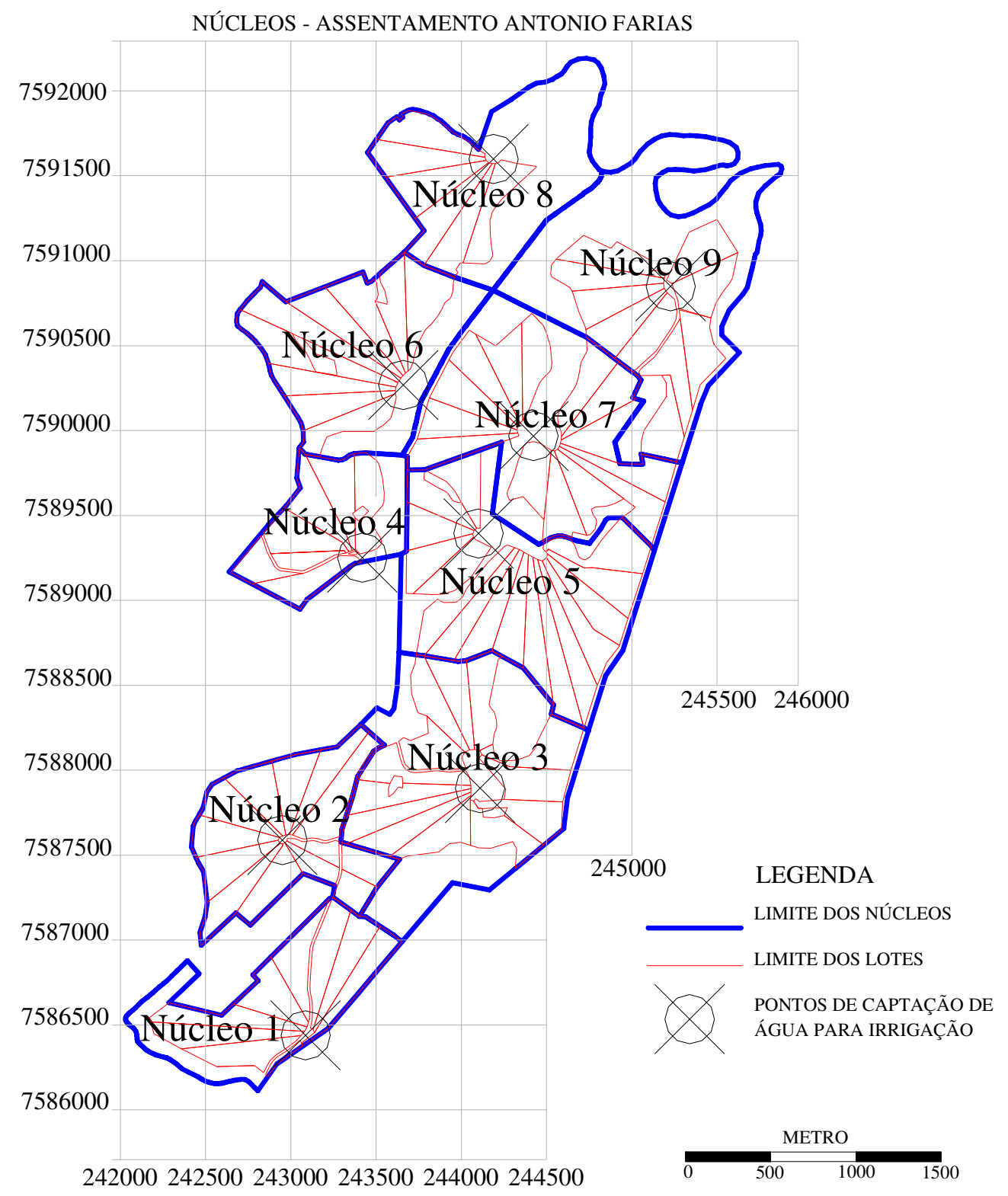

FIGURA 2. Núcleos do assentamento Antônio Farias e localização dos pontos de captação de água para irrigação.

Conforme BERNARDO (1995), e adotando o método de irrigação localizada, a lâmina real necessária a ser aplicada por irrigação, em relação à área total, foi calculada pela equação:

$\mathrm{IRN}=\mathrm{ET}_{\mathrm{L}} \mathrm{TR}$

em que,

IRN - irrigação real necessária, mm;

$\mathrm{ET}_{\mathrm{L}}$ - evapotranspiração no sistema localizado, $\mathrm{mm} \mathrm{dia}^{-1}, \mathrm{e}$

TR - intervalo entre irrigações (turno de rega), dias.

Neste trabalho, a estimativa da IRN foi baseada no maior déficit observado ao longo de todo o ciclo de cada cultura. Como a evapotranspiração normalmente é expressa em termos de lâmina d'água evaporada por dia em toda a área irrigada, sendo que em sistemas localizados não se molha toda a área 
irrigada, foi considerada uma percentagem da área molhada (P) para a cultura do coqueiro de $60 \%$, conforme recomendação técnica usualmente adotada na região.

Considerando os valores de coeficiente de cultura $(\mathrm{kc})$ apresentados por DOORENBOS \& KASSAM (1994) e PEREIRA \& ALLEN (1997), a evapotranspiração potencial da cultura foi estimada em $5,4 \mathrm{~mm} \mathrm{~d}^{-1}$, caracterizando o período de maior demanda evapotranspirométrica ao longo do ciclo da cultura.

A fim de possibilitar o cálculo da vazão de projeto (Q), foram necessárias as estimativas do tempo de funcionamento por posição (T), conforme eq.(2).

$$
\mathrm{T}=\frac{\mathrm{ITN} \mathrm{A}_{1}}{\mathrm{n} \mathrm{q}} \text { (para irrigação por árvore) }
$$

em que,

ITN - irrigação total necessária, mm;

$\mathrm{A}_{1}$ - área representada por cada árvore;

$\mathrm{n}$ - número de emissores, $\mathrm{L} \mathrm{h}^{-1}$, e

q - vazão do emissor, $\mathrm{L} \mathrm{h}^{-1}$.

Para a realização deste estudo, além dos já citados, foram adotados os parâmetros TR de 1 dia e área irrigada de 1 ha.

A capacidade do sistema, expressa pela vazão de projeto ( $\mathrm{Q}$ em $\left.\mathrm{Ls}^{-1}\right)$, foi determinada pela expressão (BERNARDO, 1995):

$$
\mathrm{Q}=2,78 \frac{\mathrm{A} \mathrm{ET}_{\mathrm{L}} \mathrm{Tr}}{\mathrm{Nu} \mathrm{TEa}}=2,78 \frac{\mathrm{A} \mathrm{ITN}}{\mathrm{NuT}}
$$

em que,

A - área do projeto, ha;

Ea - eficiência de aplicação do sistema de irrigação, decimal, e

Nu é o número de unidades operacionais em que o sistema é dividido.

\section{Cálculo da altura manométrica e potência necessária}

A altura manométrica de uma instalação elevatória $\left(\mathrm{H}_{\mathrm{m}}\right)$ é calculada pela expressão:

$$
\mathrm{H}_{\mathrm{m}}=\mathrm{H}_{\mathrm{G}}+\mathrm{hf}_{\mathrm{T}}+\mathrm{P}
$$

sendo,

$\mathrm{H}_{\mathrm{G}}$ - altura geométrica da instalação (altura de sucção + altura de recalque);

$\mathrm{hf}_{\mathrm{T}}$ - perda de carga total da tubulação, mca, e

P - Pressão necessária ao projeto, mca.

Para o cálculo da perda de carga nas tubulações $\left(\mathrm{hf}_{\mathrm{T}}\right)$, foi utilizada a fórmula de Hazen-Williams, de uso generalizado na literatura (NEVES, 1979):

$$
\mathrm{hf}_{\mathrm{T}}=\frac{10,646 \mathrm{Q}^{1,85}}{\mathrm{C}^{1,85} \mathrm{D}^{4,87}} \mathrm{~L}^{\prime}
$$

em que,

D - diâmetro da tubulação, m;

$\mathrm{Q}$ - vazão, $\mathrm{m}^{3} \mathrm{~s}^{-1}$;

$\mathrm{L}^{\prime}$ - comprimento virtual da tubulação, m, e

C - coeficiente que depende da natureza das paredes e estado de conservação da tubulação. 
$\mathrm{Na}$ eq.(5), o valor do coeficiente $\mathrm{C}$ foi considerado 140, o qual corresponde a condutos de PVC normalmente utilizados para irrigação e, por se tratar de pequenos projetos, para o cálculo de $\mathrm{H}_{\mathrm{m}}$ foi utilizado um diâmetro de tubulação de $50 \mathrm{~mm}$, devido à necessidade de trabalhar com PN 80, uma vez que os cálculos preliminares mostraram que, em alguns pontos, tubulações com PN 40 não suportariam a pressão. Além disso, esse diâmetro é comumente utilizado em pequenos projetos de irrigação. Foi fixado valor de $10 \%$ do comprimento real da tubulação como sendo valor fictício correspondente à perda de carga localizada. Portanto, com o cálculo da vazão utilizando a eq.(3), a estimativa da perda de carga nas tubulações (ht) ficou condicionada a distância do projeto à fonte d'água (L).

Com o cálculo da altura manométrica, a potência do sistema foi estimada pela equação (CARVALHO, 1984):

$$
\mathrm{Pot}=\frac{\gamma \mathrm{Q} \mathrm{Hm}}{75 \eta}
$$

em que,

Pot - potência requerida pela bomba, cv;

$\gamma$ - peso específico do líquido bombeado (água $=101,94 \mathrm{~N} \mathrm{~m}^{-3}$ ), e

$\eta$ - rendimento da bomba, decimal.

A potência do motor ou da placa $(\mathrm{N})$ é a própria potência requerida pelo sistema acrescida de uma folga, a fim de proteger o sistema contra eventuais sobrecargas. Para isso, foram utilizados os valores apresentados por DENÍCULI (1994), ou seja, para motores de até 1,47 kW (2 cv), a folga é de $50 \%$; se a potência requerida pela bomba estiver entre 1,47 e $3,68 \mathrm{~kW}$ ( 2 e $5 \mathrm{cv}$, respectivamente), a folga deve ser de $30 \%$; entre 3,68 e $7,35 \mathrm{~kW}$ (5 e $10 \mathrm{cv}$, respectivamente), a folga é de $20 \%$.

\section{Desembolsos com a compra da tubulação e da motobomba}

O preço adotado para o metro de tubo de $50 \mathrm{~mm}$ PN 80 foi de $\mathrm{R} \$ 3,00$, conforme consulta comercial. Na Tabela 1, apresenta-se o custo médio de motobombas centrífugas encontradas no mercado, em função da potência requerida.

TABELA 1. Custo médio de motobombas centrífugas em função da potência.

\begin{tabular}{cc}
\hline Potência kW $(\mathrm{cv})$ & Custo* $(\mathrm{R} \$)^{*}$ \\
\hline $0,18(0,25)$ & 135,00 \\
$0,24(0,33)$ & 141,00 \\
$0,37(0,5)$ & 155,00 \\
$0,55(0,75)$ & 162,00 \\
$0,73(1,0)$ & 271,00 \\
$1,10(1,5)$ & 340,00 \\
$1,47(2,0)$ & 470,00 \\
$2,21(3,0)$ & 534,00 \\
$3,68(5,0)$ & $1.390,00$ \\
$5,51(7,5)$ & $1.764,00$ \\
$7,35(10,0)$ & $1.886,00$ \\
$9,19(12,5)$ & $2.084,00$ \\
$11,03(15,0)$ & $2.364,00$ \\
\hline
\end{tabular}

* Valor de referência US\$1.0 = R \$ 3,689 (3-12-2002) 


\section{Demais critérios hidráulicos}

Neste estudo, foi adotado o sistema de irrigação por gotejamento. A pressão estimada e necessária ao projeto (variável P da eq.(4)) e a vazão adotada para os gotejadores foram de $200 \mathrm{kPa}$ e $0,004 \mathrm{~m}^{3} \mathrm{~h}^{-1}\left(3,0 \mathrm{~L} \mathrm{~h}^{-1}\right)$, respectivamente. Foram ainda considerados: uma unidade operacional e quatro gotejadores por planta. Portanto, o tempo de funcionamento calculado pela eq.(2) foi de 12,65 horas por posição e a vazão necessária ao projeto [eq.(3)] foi de $0,79 \mathrm{~L} \mathrm{~s}^{-1}$. O rendimento adotado para os sistemas de irrigação foi de $90 \%$ e da motobomba de $60 \%$.

\section{Obtenção dos mapas-base}

Para a área estudada, foi utilizado mapa de solos em formato digital, fornecido pelo ITERJ, na escala de 1:15.000. Em seguida, esse mapa, em formato vetorial, foi importado pelo SIG IDRISI $3.2 \mathrm{e}$ convertido para o formato matricial, gerando mapa temático com as respectivas classes de solos, conforme Figura 3.

No processo de "rasterização", transformação do formato vetorial para o matricial, foi utilizado o sistema de projeção utm (universal transverse mercator), considerando o fuso $24 \mathrm{~s}$. Os mapas foram gerados com resolução de $2 \mathrm{~m}$ x $2 \mathrm{~m}$, perfazendo 2.500 linhas por 2.500 colunas. No software IDRISI 32, esse procedimento é realizado criando-se, inicialmente, uma imagem de fundo, por meio do módulo INITIAL. Esse mesmo procedimento foi utilizado na criação dos mapas de hidrografia e curva de nível, mantendo com isso a mesma resolução. Em seguida, o módulo POLYRAS foi utilizado a fim de possibilitar efetivamente a criação dos mapas de solos, conforme Figura 3.

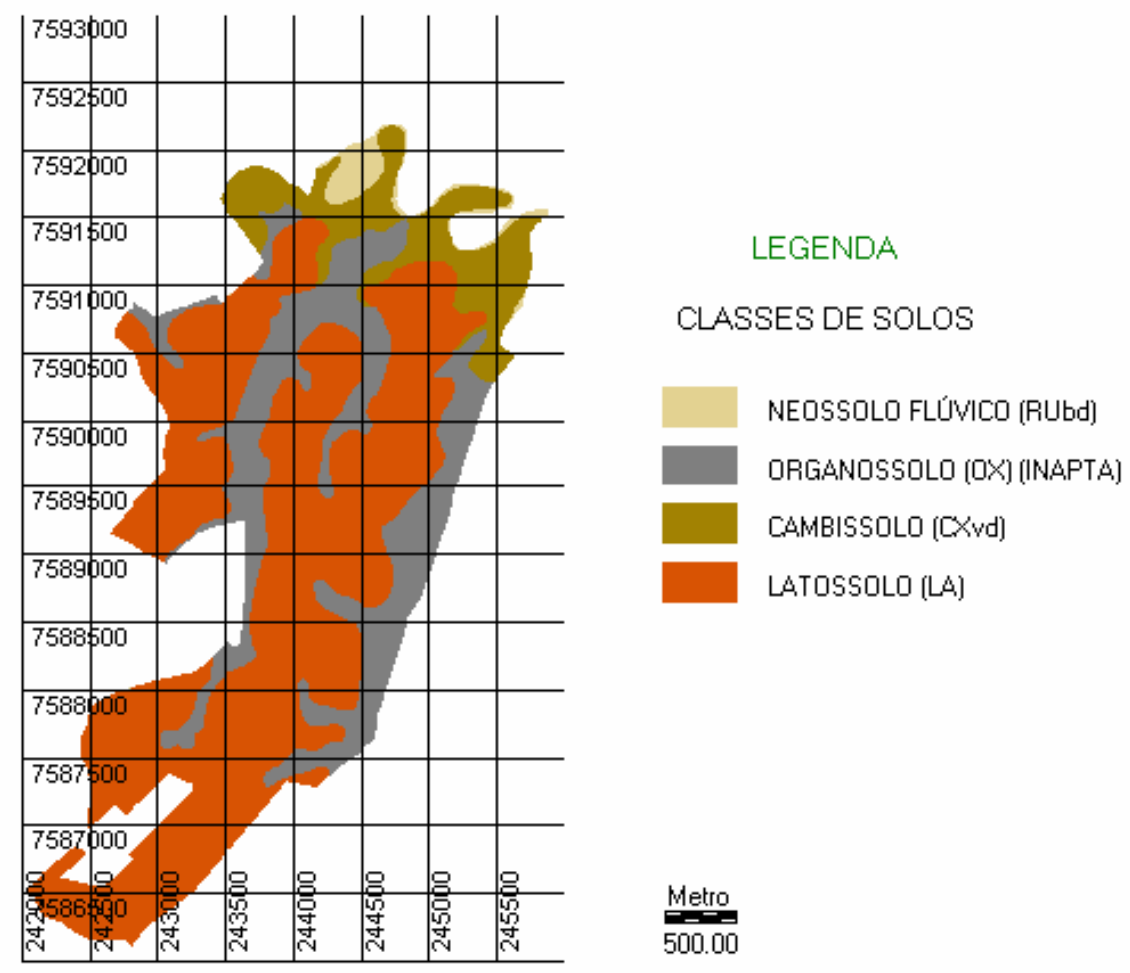

FIGURA 3. Mapa de solos do assentamento Antônio Farias.

A reclassificação do mapa de solos foi realizada atribuindo-se pesos correspondentes ao custo necessário para atender às exigências de calagem e de adubação para o plantio da cultura do coqueiro. $\mathrm{Na}$ Tabela 2, apresentam-se as necessidades de calagem e adubação de cada unidade de mapeamento para a área estudada e os seus respectivos desembolsos (pesos) necessários à correção do solo. 
Após reclassificação, o mapa gerado serviu de base para a geração do mapa de aptidão agroeconômica para a irrigação da cultura do coqueiro.

TABELA 2. Desembolso total $\left(\mathrm{R} \$ \mathrm{ha}^{-1}\right)$ para compra de calcário e fertilizantes necessários para a correção do solo para a cultura do coqueiro, de acordo com os tipos de solo do assentamento.

\begin{tabular}{|c|c|c|c|c|c|c|c|}
\hline \multirow{2}{*}{ Tipo de Solo } & \multicolumn{4}{|c|}{ Necessidade de Adubação (kg ha $\left.{ }^{-1}\right)$} & \multirow{2}{*}{$\begin{array}{c}\text { Custo Calagem } \\
\mathrm{R} \$ \mathrm{ha}^{-1}\end{array}$} & \multirow{2}{*}{$\begin{array}{l}\text { Custo Adubação } \\
\mathrm{R} \$ \mathrm{ha}^{-1}\end{array}$} & \multirow{2}{*}{$\begin{array}{l}\text { Custo } \\
\text { Total }\end{array}$} \\
\hline & Calagem $\left(\mathrm{t} \mathrm{ha}^{-1}\right)$ & $\mathrm{N}$ & $\mathrm{P}$ & $\mathrm{K}$ & & & \\
\hline Latossolo & 3,28 & 40 & 800 & 280 & 360,80 & 690,00 & $1.050,80$ \\
\hline Neossolo Flúvico & - & 25 & 400 & - & - & 510,00 & 510,00 \\
\hline Cambissolo & - & 25 & 400 & 125 & - & 660,00 & 660,00 \\
\hline
\end{tabular}

Calcário dolomítico PRNT 80\% = R \$ 110,00 por tonelada (frete incluído)

\section{Criação do Modelo Digital de Elevação (MDE), identificação dos pontos de captação e mapa de faixas equiidistantes}

Da mesma forma como realizado para os mapas de solos, o mapa de curvas de nível (planialtimétricos) foi digitalizado com resolução de 300 dpi no formato A0. O arquivo de imagem (TIF) foi importado pelo software TOPOEVN 5.0, gerando arquivos vetoriais em formato DXF (Drawing interchange file). Em seguida, esses arquivos foram também importados pelo IDRISI 32, utilizando, entretanto, o módulo LINERAS, após o procedimento básico de criação da imagem fundo (INITIAL).

Para a confecção do MDE, foi utilizado o módulo INTERCON para interpolação das curvas de nível, gerando um modelo digital de elevação do terreno, definido como qualquer representação digital da variação contínua do relevo no espaço (BURROUGHT, 1990).

Com relação aos pontos de captação (Figura 2), os mesmos tiveram suas coordenadas geográficas identificadas e assim foi criado um arquivo vetorial que, exportado para o IDRISI, foi convertido em formato matricial utilizando o módulo POINTRAS. A partir do mapa dos pontos de captação de água para irrigação, foi utilizado o módulo DISTANCE, a fim de criar faixas eqüidistantes a partir dos mesmos.

\section{Mapas de Altura Geométrica (Hg) e perda de carga (Ht)}

O mapa de altura geométrica foi obtido subtraindo-se o valor da cota de cada ponto de captação de água do modelo digital de elevação (MDE). Para essa operação, trabalhou-se com o operador matemático do IDRISI 3.2 chamado de SCALAR, que permite realizar diversas operações matemáticas em mapas.

Substituindo, na eq.(5), o valor do comprimento virtual das tubulações (L`) pelo mapa de faixas eqüidistantes dos pontos de captação para irrigação gerado pelo módulo DISTANCE e utilizando-se do operador matemático SCALAR do IDRISI 32, foi possível gerar o mapa de perda de carga, que apresenta valores mais altos à medida que se afasta dos pontos de captação de água.

\section{Mapas de altura manométrica $(\mathrm{Hm})$ e potência (Pot)}

O mapa de altura manométrica foi obtido somando-se o mapa de perda de carga (Ht) com o mapa de altura geométrica $(\mathrm{Hg})$, utilizando o módulo OVERLAY do IDRISI 32. Com isso, para cada assentamento, foi obtido o mapa $(\mathrm{Hg}+\mathrm{Ht})$ para cada ponto de captação de água para irrigação. Esse foi, então, somado ao valor da pressão do sistema adotado (20 mca), gerando-se, dessa forma, o mapa da altura manométrica referente a cada ponto de captação.

Para a criação do mapa de potência requerida pelas bombas, o mapa de altura manométrica foi multiplicado pelo valor numérico obtido a partir da eq.(6), utilizando-se do módulo SCALAR do 
IDRISI 3.2, gerando-se, dessa forma, o mapa de potência para cada ponto de captação. Esse mapa foi reclassificado, utilizando o módulo RECLASS, em classes correspondentes aos valores recomendados de folga para motores elétricos.

\section{Mapas de custo de implantação da irrigação ( CI )}

O mapa de custo de implantação da irrigação foi criado somando-se os mapas de custo de bombeamento com os mapas de custo de tubulação. Essa operação foi feita utilizando-se do módulo OVERLAY do IDRISI 3.2.

O mapa de custo das motobombas foi criado multiplicando-se o mapa de potência pelo valor correspondente ao custo da motobomba, em função da potência, apresentados na Tabela 1.

Para a criação do mapa de custo com tubulação, multiplicou-se o mapa de faixas eqüidistantes dos pontos de captação pelo custo da tubulação $\left(\mathrm{R} \$ \mathrm{~m}^{-1}\right)$, gerando-se, dessa maneira, mapas que mostram o gasto com tubulação para conduzir a água dos pontos de captação até o local de aplicação.

\section{Mapa do desembolso total}

O mapa de custo total (gastos com compra de calcário, fertilizantes, tubulação e motobomba) para a implantação da cultura do coqueiro foi obtido somando-se os mapas de custos total da irrigação com os mapas de custos de adubação e calagem. Foi possível, dessa maneira, gerar, para cada núcleo que compõe a área estudada, mapas que mostram o custo necessário para implantar 1 ha irrigado em função dos parâmetros hidráulicos e agronômicos adotados.

O mapa de custo de adubação e calagem foi obtido a partir de cálculos baseados nas análises químicas de fertilidade dos solos das duas áreas estudadas (Tabela 2). Para cada unidade de mapeamento, foi atribuído o valor necessário para adubar e corrigir a acidez do solo conforme necessidade da cultura. Esse valor constitui o peso utilizado para reclassificação em cada unidade.

\section{RESULTADOS E DISCUSSÃO}

Em função do grande número de mapas gerados para atingir o objetivo do trabalho, serão apresentados e discutidos apenas os mapas finais de custo de implantação do coqueiro-anão irrigado. Nas Figuras 4 a 8, esses mapas estão representados para os núcleos de 1 a 9 do assentamento Antônio Farias.

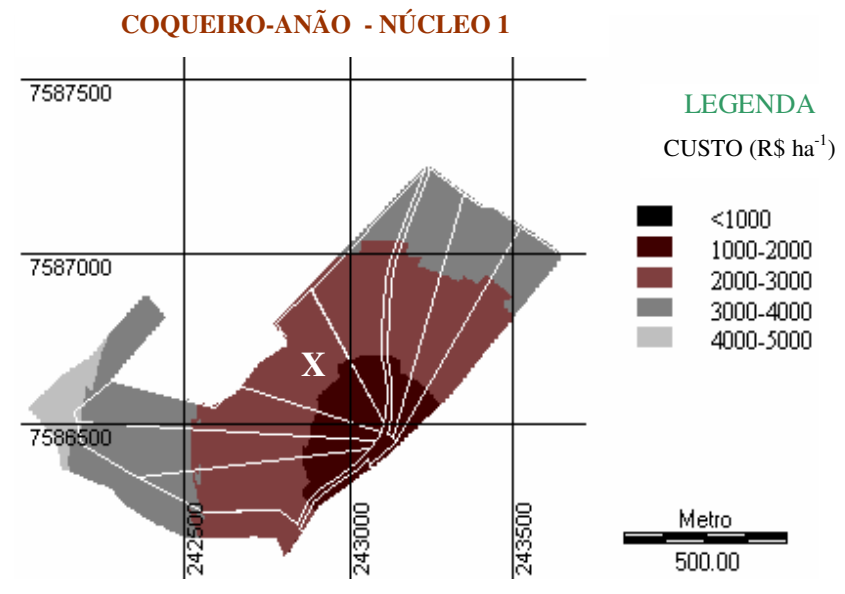

a

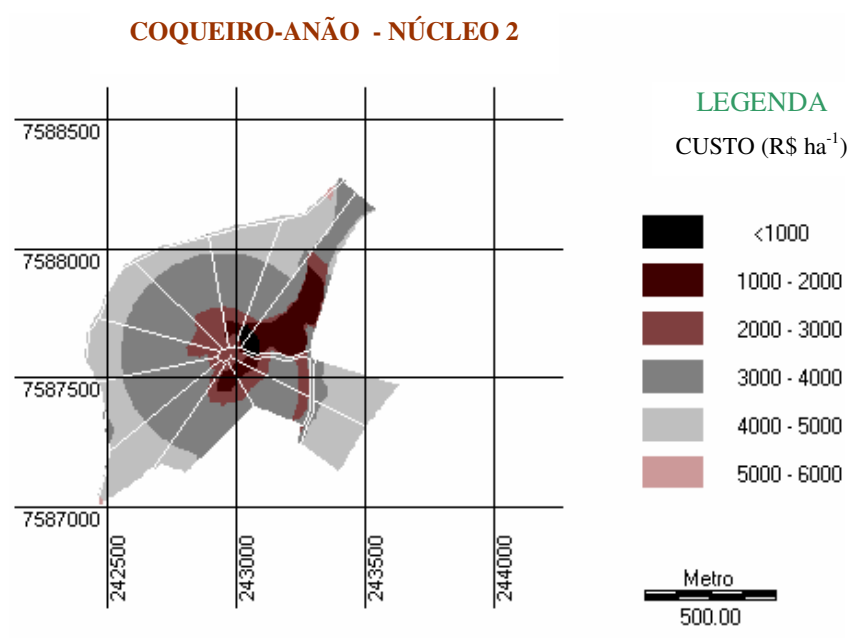

b

FIGURA 4. Custos de implantação $\left(\mathrm{R} \$ \mathrm{ha}^{-1}\right)$ da cultura do coqueiro-anão irrigado para os núcleos 1 (a) e 2 (b) do Assentamento Antônio Farias. 


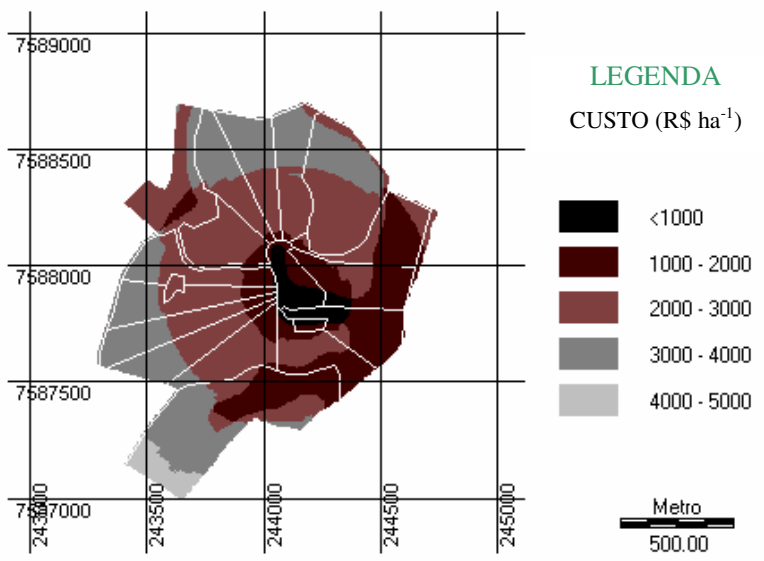

a

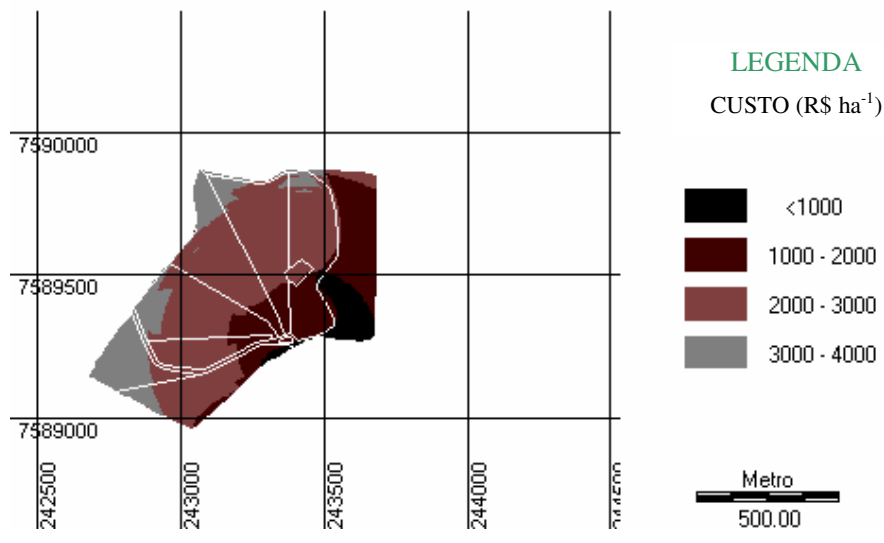

b

FIGURA 5. Custos de implantação $\left(\mathrm{R} \$ \mathrm{ha}^{-1}\right)$ da cultura do coqueiro-anão irrigado para os núcleos 3 (a) e 4 (b) do Assentamento Antônio Farias.

COQUEIRO-ANÃO - NÚCLEO 5

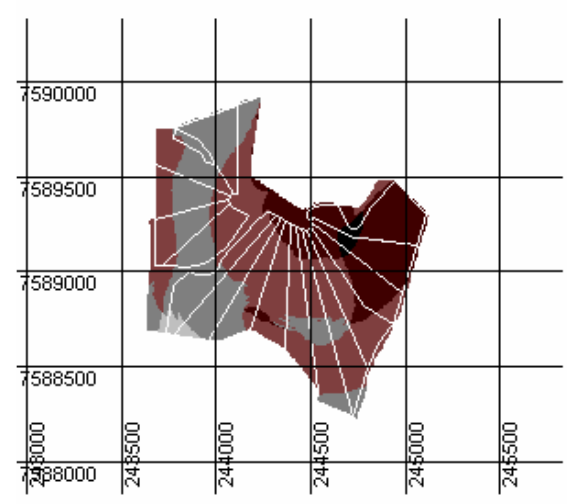

a

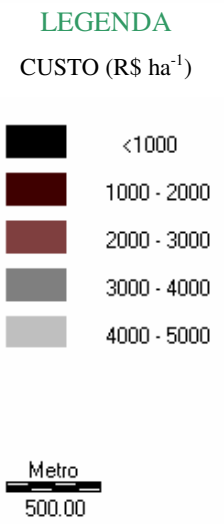

LEGENDA

$000 \cdot 2000$

3000

$000 \cdot 4000$

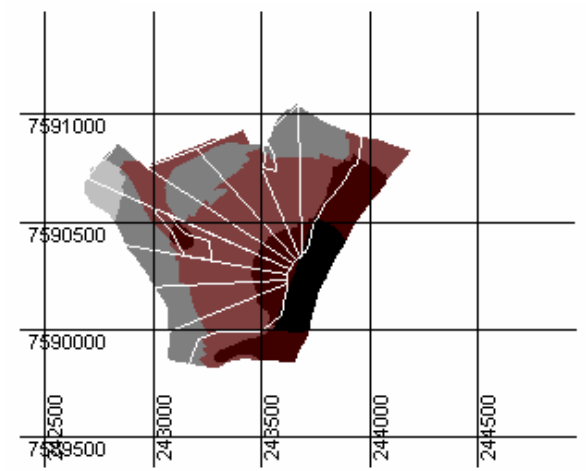

LEGENDA

CUSTO $\left(\mathrm{R} \$ \mathrm{ha}^{-1}\right)$

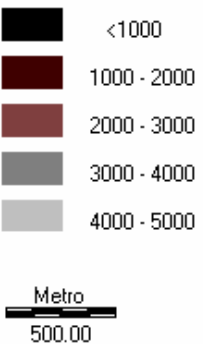

b

FIGURA 6. Custos de implantação $\left(\mathrm{R} \$ \mathrm{ha}^{-1}\right)$ da cultura do coqueiro-anão irrigado para os núcleos 5 (a) e 6 (b) do Assentamento Antônio Farias.

COQUEIRO-ANÃO - NÚCLEO 7

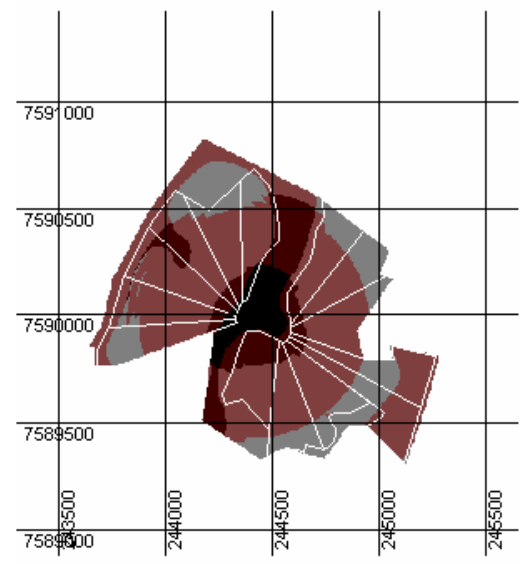

a
COQUEIRO-ANÃO - NÚCLEO 8

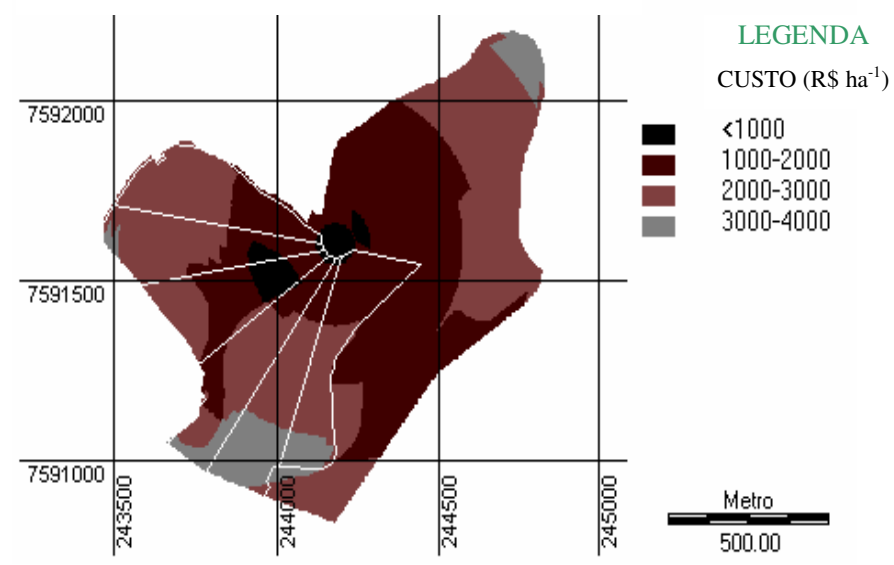

b

FIGURA 7. Custos de implantação $\left(\mathrm{R} \$ \mathrm{ha}^{-1}\right)$ da cultura do coqueiro-anão irrigado para os núcleos 7 (a) e 8 (b) do Assentamento Antônio Farias. 


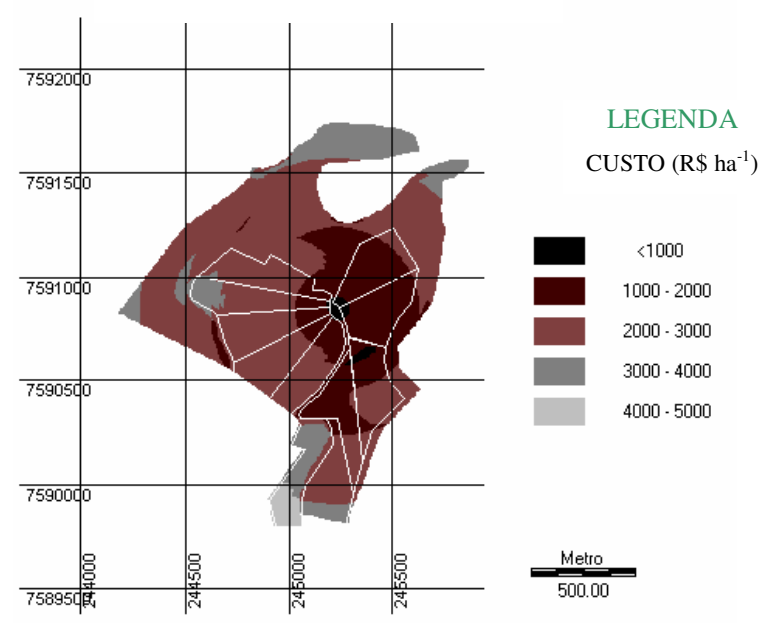

FIGURA 8. Custos de implantação $\left(\mathrm{R} \$ \mathrm{ha}^{-1}\right)$ da cultura do coqueiro-anão irrigado para o núcleo 9 do Assentamento Antônio Farias.

Observando os mapas, percebe-se que o custo de implantação do coqueiro irrigado no assentamento rural Antônio Farias atingiu as faixas de 3.000,00 a 4.000,00 R \$ ha ${ }^{-1}$ para os núcleos 4; 7 e 8; e de 4.000,00 a 5.000,00 $\mathrm{R} \$$ ha $^{-1}$ para os núcleos $1 ; 3 ; 5 ; 6$ e 9. Para o núcleo 2, a faixa mais alta de custo atingiu a classe de 5.000,00 a 6.000,00 $\mathrm{R} \$ \mathrm{ha}^{-1}$. Em função dos parâmetros necessários para a obtenção dos mapas, esses valores indicam como os parâmetros solo e distância do ponto de captação, dentre outros, interferem no custo de implantação.

Nas figuras, constata-se, também, que a hipótese de que os sistemas de informações geográficas podem ser utilizados como ferramenta na identificação de áreas com diferentes potenciais agroeconômicos é verdadeira. Ainda para completar as informações referentes às Figuras, na Tabela 3, apresentam-se as porcentagens de área ocupada em cada classe de custo de implantação, para os núcleos desse assentamento.

Apesar de terem sido observadas classes de custo de implantação elevadas, percebe-se, com os dados da Tabela 3, que custos inferiores a $\mathrm{R} \$ 3.000,00$ por hectare são observados em mais de $60 \%$ da área útil para os núcleos $1 ; 3 ; 5 ; 6$ e 7. Para os núcleos $4 ; 8$ e 9, custos inferiores a esse valor são observados em mais de $80 \%$ da área útil irrigável. Esse resultado é distinto para o núcleo 2, que apresentou até essa classe de custo, apenas 17,3\% de sua área útil. Percebe-se que esse núcleo apresentou a classe de custo de $\mathrm{R} \$ 5.000,00$ a $\mathrm{R} \$ 6.000,00$ por hectare em apenas 0,2\% de sua área. De qualquer forma, esse núcleo, em função de suas características, apresentou o maior custo para a implantação da agricultura irrigada.

TABELA 3. Porcentagem das áreas por classe de custo de implantação para a cultura do coqueiroanão, para o Assentamento Antônio Farias.

\begin{tabular}{|c|c|c|c|c|c|c|c|c|c|}
\hline \multirow{2}{*}{$\begin{array}{c}\text { Custo } \\
\left(\mathrm{R} \$ \mathrm{ha}^{-1}\right)\end{array}$} & \multicolumn{9}{|c|}{ Núcleo } \\
\hline & 1 & 2 & 3 & 4 & 5 & 6 & 7 & 8 & 9 \\
\hline $0-1.000$ & - & 0,81 & 3,88 & 0,83 & 1,24 & 0,14 & 0,80 & 4,33 & 1,01 \\
\hline $1.000-2.000$ & 16,05 & 7,01 & 22,45 & 16,14 & 22,86 & 10,45 & 9,98 & 30,68 & 24,23 \\
\hline $2.000-3.000$ & 52,30 & 9,47 & 42,37 & 63,68 & 44,61 & 50,09 & 67,60 & 52,56 & 59,23 \\
\hline $3.000-4.000$ & 31,43 & 44,57 & 28,89 & 19,34 & 30,26 & 34,89 & 21,63 & 12,43 & 13,90 \\
\hline $4.000-5.000$ & 0,22 & 37,95 & 2,42 & - & 1,02 & 4,43 & - & - & 1,63 \\
\hline $5.000-6.000$ & - & 0,20 & - & - & - & - & - & - & - \\
\hline Área total (ha) & 63,92 & 81,93 & 152,22 & 45,65 & 121,23 & 78,18 & 100,89 & 47,07 & 153,85 \\
\hline
\end{tabular}


Com o objetivo de melhor aprofundar a discussão desses resultados, foi realizada análise detalhada entre os núcleos 1 e 8 , por apresentarem resultados bem contrastantes no planejamento da cultura do coqueiro irrigado. Observa-se que o núcleo 1 apresenta área proporcionalmente maior ocupada com faixas de custo mais elevada em relação ao núcleo 8. Observando a Tabela 3, nota-se que, para o núcleo $1 ; 16,05 \%$ da área apresenta custo entre $\mathrm{R} \$ 1.000,00$ e $\mathrm{R} \$ 2.000,00$ por hectare. Em $52,30 \%$ da área, os custos ficaram entre $\mathrm{R} \$ 2.000,00$ e $\mathrm{R} \$ 3.000,00$ por hectare; em 31,43\%, os custos foram de $\mathrm{R} \$ 3.000,00$ a $\mathrm{R} \$ 4.000,00$ por hectare e, em 0,22\% da área, os custos ficaram entre $\mathrm{R} \$$ 4.000,00 e R \$ 5.000,00 por hectare. Com relação ao núcleo 8; 4,33\% da área apresenta custo menor do que $\mathrm{R} \$ 1.000,00$ por hectare. Em 30,68\% da área, os custos ficaram entre $\mathrm{R} \$ 1.000,00$ e $\mathrm{R} \$ 2.000,00$ por hectare; em 52,56\% da área, os custos foram de $\mathrm{R} \$ 2.000,00$ a $\mathrm{R} \$ 3.000,00$ por hectare e, em $12,43 \%$ da área, os custos ficaram entre $\mathrm{R} \$ 3.000,00$ e $\mathrm{R} \$ 4.000,00$ por hectare.

A variação no custo de implantação entre os núcleos 1 e 8 ocorreu principalmente devido ao custo de adubação e calagem. O núcleo 1 está totalmente localizado sobre um Latossolo (Figura 9), que possui baixa fertilidade e $\mathrm{pH}$ mais elevado (Tabela 2), o que contribuiu para elevar o custo final de implantação da cultura. No núcleo 8, o valor de correção e adubação do solo foi menor, pois está localizado sobre manchas de diferentes solos (Figura 10) e que apresentam menores necessidades de adubação e calagem.

\section{SOLOS - NÚCLEO 1 - COQUEIRO-ANÃO}

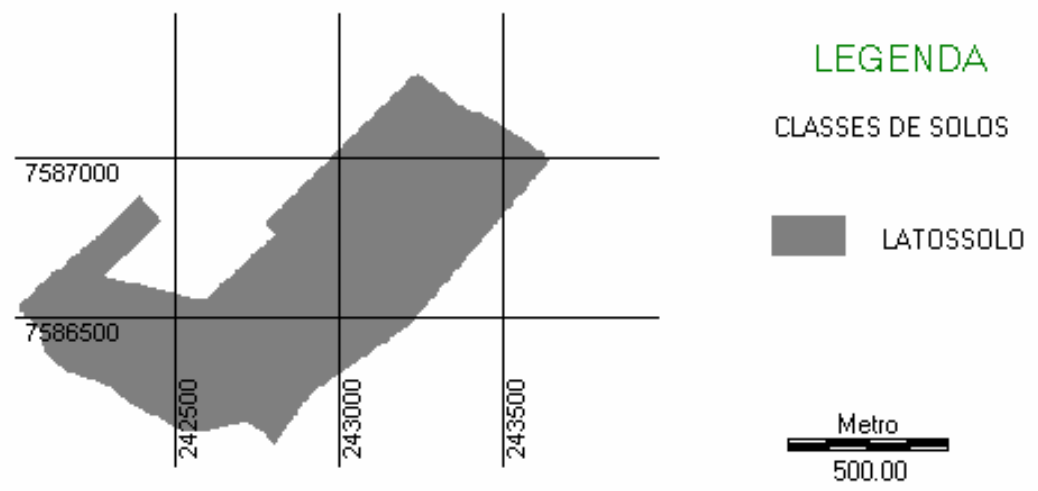

FIGURA 9. Mapa de solo referente ao núcleo 1 do Assentamento Antônio Farias.

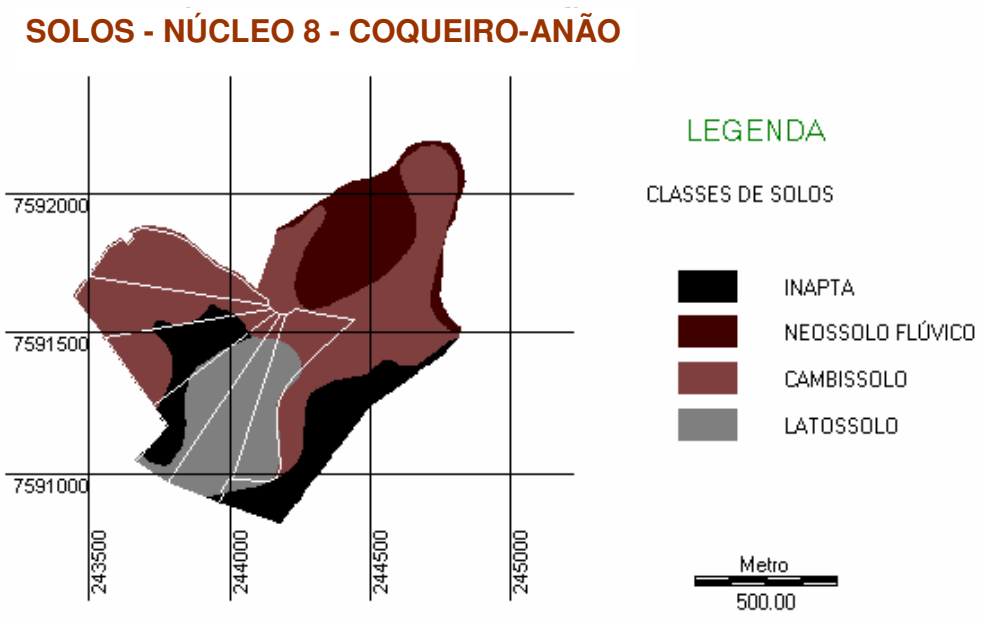

FIGURA 10. Mapa de solo referente ao núcleo 8 do Assentamento Antônio Farias. 
Além disso, os lotes pertencentes ao núcleo 1 possuem maior comprimento, apresentando faixas mais distantes do ponto de captação. Esse fato faz com que o comprimento da tubulação e, consequientemente, a perda de carga e a potência necessária ao conjunto motobomba sejam maiores (Figura 11). Em contrapartida, os lotes do núcleo 8 são mais curtos, ficando mais próximos do ponto de captação (Figura 12).

FAIXAS EQÜIDISTANTES - NÚCLEO 1

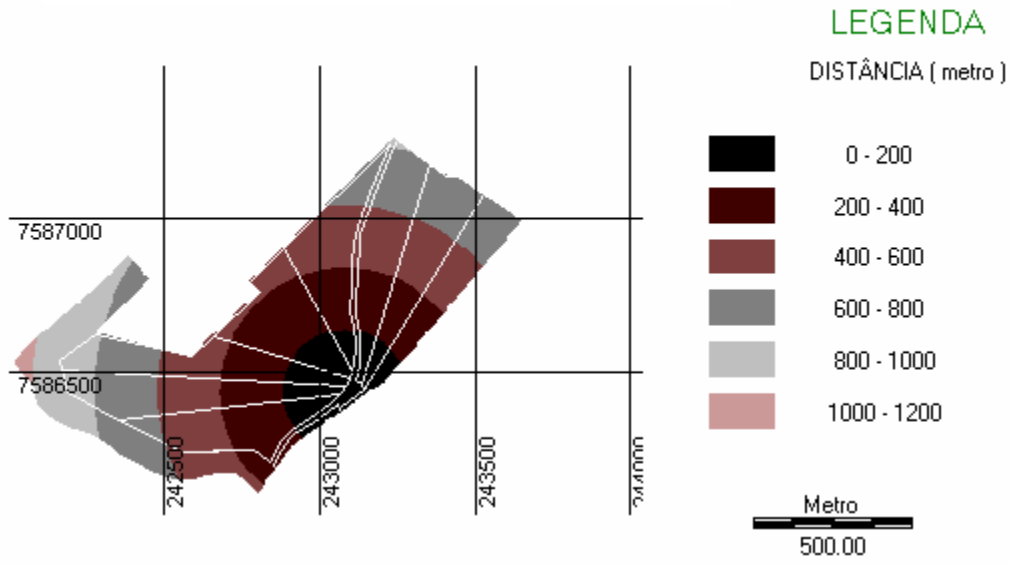

FIGURA 11. Mapa de faixas eqüidistantes dos pontos de captação de água para irrigação, referente ao núcleo 1 do Assentamento Antônio Farias.

\section{FAIXAS EQÜIDISTANTES - NÚCLEO 8}

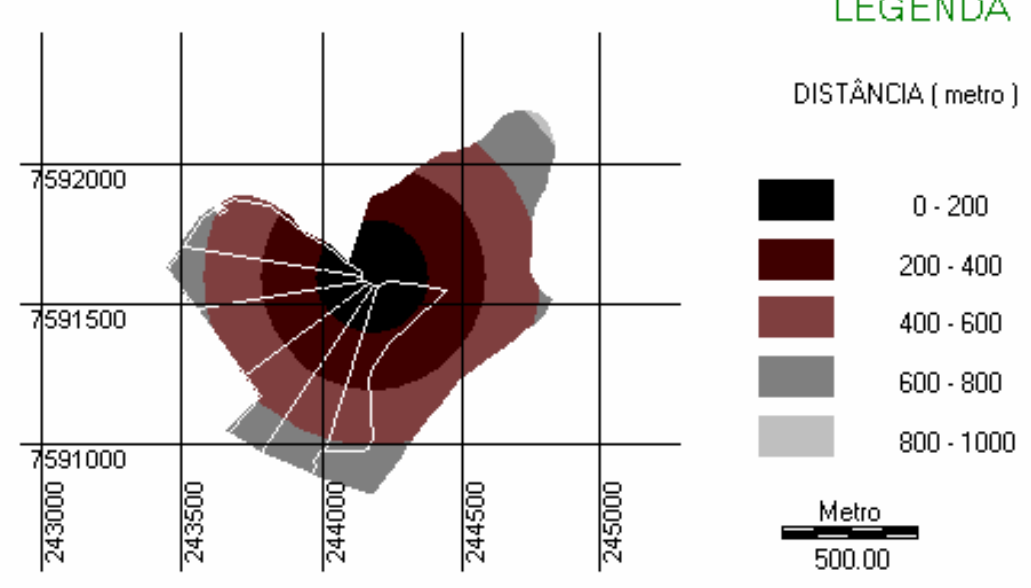

FIGURA 12. Mapa de faixas eqüidistantes dos pontos de captação de água para irrigação, referente ao núcleo 8 do Assentamento Antônio Farias.

Percebeu-se ainda, que, dentro de cada núcleo, os custos de implantação foram diferentes, mostrando que existem lotes que se encontram em áreas privilegiadas, onde o custo para implantar 1 ha de coqueiro-anão irrigado é menor quando comparado com outros lotes dentro do mesmo núcleo. Avaliando o núcleo 1, pode-se notar, na Figura 4a , que o lote identificado pela letra "x" é melhor agroeconomicamente se comparado com os demais lotes, pois apresenta menores custos de implantação, com maior porcentagem de área localizada nas classes de $\mathrm{R} \$ 1.000,00$ a $\mathrm{R} \$ 3.000,00$ por hectare. Além disso, esse mesmo lote apresenta mais de $50 \%$ de sua área na classe de $\mathrm{R} \$ 2.000,00$ a $\mathrm{R} \$ 3.000,00$ por hectare. 


\section{CONCLUSÕES}

A metodologia utilizando o SIG mostrou-se eficaz na obtenção de informações necessárias ao planejamento de áreas irrigadas.

Os sistemas de informações geográficas foram capazes de gerar informações, apresentadas na forma de mapas, que permitiram identificar regiões mais aptas agroeconomicamente para a implantação da cultura estudada, a partir da sobreposição de informações digitais de solo, clima e relevo, principalmente.

O custo de implantação das culturas estudadas foi mais influenciado pelo custo da tubulação (devido a distância até o ponto de captação) e também pelo custo da motobomba do que pelo custo da adubação e calagem.

As informações geradas neste estudo mostraram que existem variações significativas nos gastos necessários para implantar 1 ha de coqueiro-anão irrigado, entre os lotes dos assentados.

\section{REFERÊNCIAS}

ASSAD, E.D.; SANO, E.E. Sistemas de informações geográficas: aplicações na agricultura. Brasília: EMBRAPA-CPAC, 1993. 2.744 p.

ASSAD, E.D.; SANO, E.E. Sistemas de informações geográficas: aplicações na agricultura. 2.ed. Brasília: EMBRAPA-CPAC, 1998. 434 p.

BERNARDO, S. Manual de irrigação. 6.ed. Viçosa, MG: UFV, 1995. 657 p.

BOWMAN, J.A.; SIMMONS, F.W.; KIMPEL, B.C. Irrigation in midwest: Lessons from Illinois. Journal of Irrigation and Drainage Division, NewYork, v.117, n.5, p.700-15, 1991.

BURROUGH, P. Principles of geographical information Systems for land resources assessment. 1990. 194 f. Monographs (Soil and Resourses Survey) - Oxford University, Great Britain, 1990.

CARVALHO, D.F.; SOARES, A.A.; SEDIYAMA, G.C. Programa computacional para a estimativa da demanda de irrigação em perímetros irrigados. Revista Engenharia Rural, Piracicaba, v.10, n.2, p.59-73, 1999.

CARVALHO, D.F. Instalações elevatórias: bombas. Belo Horizonte: FUMARC, 1984. 355 p.

DENÍCULI, W. Condutos forçados.Viçosa-MG: UFV, 1994. 389 p. (Material didático da disciplina ENG-640)

DOORENBOS, J.; KASSAM, A.H. Efeito da água no rendimento das culturas. Campina Grande: UFPB, 1994. 306 p. (Estudos FAO: Irrigação e Drenagem, 33).

NEVES, E.T. Curso de hidráulica. Porto Alegre: Editora Globo, 1979. 577 p.

PEREIRA, L.S.; ALLEN, R.G. Novas aproximações aos coeficientes culturais. Engenharia Agrícola, Jaboticabal, v.16, n.4, p.118-43, 1997.

SIFUMA, J.; GICHUKI, J. Planning irrigation cropping systems. In: NATIONAL WORKSHOP, 4., 2000, Kikuyu. Proceedings... p.263-6.

SMITH, M. Report on the expert consultation on revision of FAO methodologies for crop water requeriments. Rome: FAO, 1991. 45 p. 